TITRE: CRÉATION D'UN MATÉRIEL PÉdAgOgIQUE FAVORISANT LE DÉVELOPPEMENT DE LA LITTÉRATIE EN CINQUIĖME SECONDAIRE

Auteur(s): Marianne Roy Venne, M.Éd., Université de Sherbrooke, Enseignante au Secondaire, Commission scolaire Pierre-Neveu, ISABelle Carignan, Ph.D., Université TÉluQ, Marie-Christine BeAudry, Ph.D., Université du QuÉBEC À MontrÉAL (UQAM)

Publication: CRÉATION DE DISPOSITIFS DIDACTIQUES ET ENSEIGNEMENT-APPRENTISSAGE DIVERSIFIÉ EN LITTÉRATIE : VERS UNE VALORISATION DE LA RECHERCHE-DÉVELOPPEMENT ET DE LA RECHERCHEACTION EN ÉDUCATION

PAGES: $112-130$

DiRECTEURS: MARIE-ChRISTINE BEAUdRY, ISABELLE CARIgnAN ET FRANÇOIS LAROSE ÉdITEUR: LES ÉDITIONS DE L'UNIVERSITÉ DE SHERBROOKE, 2017

ISBN: 978-2-7622-0356-1

URI: HTTP://HDL.HANDLE.NET/11143/10128

DOI: HTTP://DX.DOI.ORG/10.17118/11143/10128 


\title{
CRÉATION D'UN MATÉRIEL PÉDAGOGIQUE FAVORISANT LE DÉVELOPPEMENT DE LA LITTÉRATIE EN CINQUIÈME SECONDAIRE
}

\author{
Marianne Roy Venne, M.Éd., Université de Sherbrooke \\ Enseignante au secondaire, Commission scolaire Pierre-Neveu \\ Isabelle Carignan, Ph.D., Université TÉLUQ \\ Marie-Christine Beaudry, Ph.D., Université du Québec à Montréal (UQAM)
}

Résumé : Cet article a pour but de présenter de façon succincte l'élaboration d'un matériel pédagogique qui a été créé pour favoriser le développement de la littératie chez les élèves de cinquième secondaire (16-17 ans). Six activités respectant les étapes d'une situation d'apprentissage et d'évaluation (SAÉ) ont été conçues pour développer les trois compétences en français, langue d'enseignement, soit « Lire et apprécier des textes variés », "Écrire des textes variés » et « Communiquer oralement selon des modalités variées ». Trois genres littéraires ont été sélectionnés pour la création du matériel : l'album, le roman et la nouvelle. D'une part, nous avons voulu innover avec l'album pour les adolescents, car il s'agit d'un genre méconnu et associé aux plus jeunes. D’autre part, le roman est un genre apprécié par les adolescents tandis que la nouvelle est un genre bref, qui peut surprendre et intéresser les lecteurs adolescents. De surcroit, le roman et la nouvelle sont deux genres prescrits par le programme de formation de l'école québécoise (PFÉQ). Enfin, le matériel pédagogique a été évalué par deux expertes du milieu scolaire qui ont dressé le portrait des points forts et des points à améliorer.

Mots-clés : matériel pédagogique, secondaire, littératie, constructivisme, socioconstructivisme et développement des compétences. 


\section{Introduction}

La recherche-développement présentée ici s'intéresse au développement des compétences en littératie ${ }^{1}$ chez des élèves de cinquième secondaire. Nous avons voulu construire du matériel pédagogique unique, motivant et novateur pour ces élèves et pouvant être adapté par les enseignants, ou, du moins, pouvant les inspirer. Nous voulions imbriquer les actes d'écrire et de communiquer oralement en les travaillant autour de la lecture.

Dans le cadre de cet article, nous présenterons la problématique de façon succincte pour ensuite faire part du cadre de référence dans lequel seront définis le constructivisme, le socioconstructivisme ainsi que la situation d'apprentissage et d'évaluation (SAÉ). Nous aborderons également les différents facteurs qui favorisent le développement de la littératie. Pour ce qui est de la méthodologie, nous parlerons du type de recherche qui a été mis sur pied, de la démarche suivie pour la création du matériel pédagogique, de la démarche adoptée pour son évaluation et du traitement des données. Puis, nous décrirons le matériel soumis à l'évaluation. Enfin, nous ferons part de l'analyse des expertes et des limites du matériel.

\section{Problématique}

Comme le soulève Blaquière (2012) dans sa recherche-développement, beaucoup de chercheurs se questionnent sur la pertinence d'enseigner à l'aide d'un manuel scolaire. Plutôt entrevu comme un guide pédagogique et un modèle adaptable par les enseignants, il semblerait que le manuel scolaire ne soit pas motivant et signifiant du point de vue des élèves et des enseignants, et que son utilisation soit très limitée d'un point de vue didactique (Lenoir, Roy, Rey et Desjardins, 2001; Lebrun, Lenoir et Desjardins, 2004). D'ailleurs, Lenoir, Roy, Rey et Desjardins (2001) préconisent plutôt l'emploi en salle de classe d'activités uniques et originales créées par l'enseignant puisque le manuel scolaire n'est pas toujours pertinent et en lien avec le programme de formation. De plus, il ne laisserait pas assez de marge de manœuvre à l'enseignant pour remanier l'activité proposée et, surtout, il ne mettrait pas nécessairement les élèves en action afin de favoriser l'échange, la réflexion, la critique, la discussion, l'analyse ainsi que le questionnement comme le veulent les approches constructiviste et socioconstructiviste. De surcroit, le sentiment d'auto-efficacité (Bandura, 2003) serait plus élevé chez un enseignant qui crée son propre matériel, puisqu'il a le sentiment de la mission accomplie en fournissant du matériel intéressant et pertinent à ses élèves, que chez un enseignant qui utilise le manuel scolaire.

1. «La littératie est en lien avec le développement, chez les individus, de l'habileté à lire, à écrire, à parler et à écouter. De plus, ces pratiques « littératiées » peuvent être utilisées dans la vie courante, à la maison, au travail, à l'école ou dans la collectivité en fonction des objectifs visés par chacun, et ce, en interaction avec ses propres valeurs et sa propre culture » (Beauregard, Carignan et Létourneau, 2011, p. 8-9). 
Par ailleurs, selon le rapport québécois du ministère de l'Éducation (Gouvernement du Québec, 2007) intitulé Apprendre à lire (action concertée pour le soutien de la recherche en lecture), les élèves à risque en littératie échouent en lecture et ne s'y intéressent pas parce qu'ils n'ont pas été incités à le faire dès leur plus jeune âge. Souvent issus de milieux pauvres culturellement et défavorisés, ces élèves ne voient pas l'intérêt de lire et ils ne s'intéressent ni à la lecture ni à l'écriture. Leur milieu familial n'a pas suffisamment valorisé la langue à leurs yeux, ce qui fait que leur réussite scolaire est souvent compromise (Gouvernement du Québec, 2007). Entre ici en jeu le rôle de l'école, même en cinquième secondaire : faire apprécier et comprendre l'importance de la langue aux élèves par l'entremise de la lecture, de l'écriture et de la communication orale. Dans le cadre de notre recherche dont fait état cet article, nous avons privilégié la lecture littéraire comme trame de fond au développement de la littératie, et ce, dans une approche basée sur la construction des savoirs pour mettre les élèves au centre de leurs apprentissages.

Bien qu'il existe déjà du matériel pour enseigner la littératie et, qu'à notre connaissance, certains enseignants aient déjà abandonné l'utilisation du manuel scolaire au profit de leur propre matériel, il n’en demeure pas moins qu'il serait intéressant de créer du matériel pédagogique qui favorise le développement de la littératie par l'entremise des approches constructiviste et socioconstructiviste en cinquième secondaire. Pour nous, construire des activités d'apprentissage n'est pas simplement créer du matériel que nous pourrons utiliser en classe : c'est remettre en question notre enseignement tout en tentant de répondre aux besoins et aux attentes des élèves dans nos classes. C'est en quelque sorte considérer un éventail de facteurs, notamment leur motivation, leurs intérêts, leurs gouts, leurs capacités, leurs compétences, pour rendre la classe dynamique et, par le fait même, notre enseignement. Nous oublions souvent que nous sommes des passeurs culturels (Zakhartchouk, 1999) et que nous éduquons et formons les générations de demain, d'où l'importance d'éveiller nos élèves à la lecture, à l'écriture et à la communication orale.

À la suite de cette courte mise en contexte, une question se dégage : quelle forme pourrait prendre un matériel pédagogique permettant de développer les compétences en littératie en cinquième année du secondaire par l'entremise des approches constructiviste et socioconstructiviste?

\section{Cadre de référence}

\section{Constructivisme et socioconstructivisme}

L'approche constructiviste a été d'abord définie par Piaget (1974) pour être ensuite, au fil du temps, reprise par plusieurs auteurs, notamment Masciotra (2007). Masciotra définit le constructivisme comme étant « une posture épistémologique qui prétend qu'une personne développe son intelligence et construit ses connaissances en action par la réflexion sur l'action et ses résultats » (2007, p. 1). Pour cette approche, il ne s'agit plus d'apprendre par coeur, mais bien de placer les élèves au centre de leurs apprentissages pour qu'ils soient actifs intellectuellement. Comme le souligne 
Legendre, « [ê]tre actif ne se limite pas à manipuler concrètement des objets. C'est d'abord être intellectuellement actif, autrement dit manipuler les objets en pensée à l'aide d'opérations mentales, et établir de multiples relations entre les objets et entre les opérations elles-mêmes » (2012, p. 209). Ainsi, pour que les élèves soient actifs cognitivement, ils doivent adapter ce qu'ils apprennent de nouveau (accommodation) à ce qu'ils connaissent déjà (assimilation) (Masciotra, 2007). En réfléchissant, en construisant des liens entre leurs connaissances antérieures et les nouvelles connaissances, et en posant des questions, les élèves sont désormais en mesure de développer cognitivement leur savoir et de construire des liens entre ce qu'ils savent déjà et ce qu'ils apprennent. L'approche constructiviste en éducation reflète bien les visées de notre projet puisqu'elle permet aux élèves d'être en questionnement et d'être confrontés au point de vue des autres, mais également à celui de l'enseignant.

Le socioconstructivisme, de son côté, abonde dans le même sens que le constructivisme, mais en proposant que les élèves interagissent lors du processus d'apprentissage (Masciotra, 2007). En effet, dans la perspective de construction sociale des savoirs telle que pensée par Vygotsky (1985), les élèves doivent interagir avec le monde qui les entoure, à savoir leur environnement physique, social et culturel. Ainsi, en étant par exemple confrontés aux représentations que se font les autres de la société et de la culture, les élèves sont à même de développer eux-mêmes leurs représentations, qu'ils viennent justifier et expliquer par les valeurs qu'ils prônent et les expérimentations qu'ils font (Masciotra, 2007). L'enseignant qui travaille en s'inspirant de l'approche socioconstructiviste fait donc en sorte de mettre quotidiennement les élèves en interaction et celui-ci participe souvent à leurs échanges et à leurs activités afin de les soutenir dans cette construction du savoir.

\section{Situation d'apprentissage et d'évaluation (SAÉ)}

Lors de l'élaboration de notre matériel pédagogique, nous avons choisi les SAÉ pour créer nos activités d'apprentissage, comme le suggère le PFÉQ. Le ministère de l'Éducation, du Loisir et du Sport (MELS) définit la SAÉ comme étant

un ensemble constitué d'une ou plusieurs tâches réalisées par l'élève en vue d'atteindre le but fixé. Elle permet à l'élève de développer et d'exercer une ou plusieurs compétences disciplinaires ou transversales et à l'enseignante d'assurer le suivi du développement des compétences dans une perspective d'aide à l'apprentissage et de s'en servir pour la reconnaissance des compétences de l'élève (Gouvernement du Québec, 2007, p. 5).

Selon Bibeau (2009), la SAÉ comporte trois temps : le temps de préparation, la phase de réalisation ou d'action en classe et la phase d'intégration. Le temps de préparation consiste à présenter aux élèves un problème qu'ils auront à résoudre et à mettre en contexte le travail qui sera réalisé. C'est à cette étape qu'ils sont appelés à activer leurs connaissances antérieures et qu'ils peuvent émettre des hypothèses, des questions quant au problème à résoudre. Lors de la phase de réalisation ou 
d'action en classe, les élèves se mobilisent pour effectuer les tâches demandées. Certaines de ces tâches peuvent être destinées à un public (par exemple, production d'un blogue pouvant être lu par l'ensemble des élèves de l'école) et elles peuvent être ponctuées de moments de rétroaction, soit de l'enseignant ou encore des autres élèves. Quant à la phase d'intégration, elle consiste à amener l'élève à prendre conscience des apprentissages réalisés, à verbaliser les méthodes de travail employées, les difficultés rencontrées, de même que ses réussites et ses résultats. Cette phase permet également à l'enseignant de réguler les apprentissages réalisés, « c'est-à-dire la prise en compte des difficultés, la rétroaction significative et la remédiation si c'est nécessaire »(Bibeau, 2009). Comme le souligne Bibeau, «l'autorégulation est également de mise afin que l'élève prenne conscience de sa démarche, de ses difficultés et de ses ressources personnelles » (2009). En questionnant les élèves, par exemple sur les démarches qu'ils ont privilégiées, l'enseignant les amène à réfléchir plus en profondeur sur les outils et les méthodes qu'ils ont déployés pour la réalisation de la SAÉ.

\section{Les facteurs qui permettent le développement de la littératie}

Pour qu'il y ait développement de la littératie à travers une activité d'enseignement- apprentissage, il faut certains facteurs. Dumais (2011), en s'inspirant des écrits de Navarro et Le Deun (2004), Makdissi, Boisclair et Sirois (2010), Royer (1995) et de Giasson et Saint-Laurent (1998), est l'un de ceux qui s'est penché sur la question. Il relève les facteurs suivants : les facteurs d'ordre motivationnel et psychoaffectif, les facteurs d'ordre social, les facteurs d'ordre culturel et, ensuite, les facteurs d'ordre cognitif et métacognitif.

Les facteurs d'ordre motivationnel et psychoaffectif renvoient directement au fait que l'élève a besoin d'un modèle et que, bien souvent, outre les parents, ce sera l'enseignant. Dumais (2011) souligne l'importance d'apprendre à lire aux élèves et à apprécier les livres et la lecture en leur lisant des histoires. Au secondaire, le rôle de modèle et de motivateur de l'enseignant est d'autant plus important que les élèves qui n’apprécient pas lire, écrire ou encore manier la langue viennent parfois d'un milieu où cela n'était pas nécessairement valorisé (Navarro et Le Deun, 2004). Plusieurs auteurs mentionnent d'ailleurs le fait qu'il est essentiel que l'enfant ait des modèles pour s'intéresser à la lecture et à l'écriture. Ces modèles doivent interagir avec lui dans son environnement, et ce, au quotidien (Burns, Espinosa et Snow, 2003; Carignan et Beauregard, 2010; Dumais, 2011). Le matériel pédagogique que nous avons créé tient compte de ces facteurs puisqu'il propose des activités où l'enseignant lit à voix haute, reflétant ainsi sa passion pour la lecture et les mots à l'ensemble de la classe. Dans une idée de partage, de coopération et d'échanges, les élèves sont aussi amenés à discuter d'une œuvre et à s'ouvrir aux autres. Nous pensons qu'il est primordial de travailler avec le concept de littératie à cet égard puisque plusieurs élèves ont de nombreuses carences en lecture et en écriture, du fait qu'ils viennent d'un milieu où cela n'était pas valorisé. Ils «n'ont pas la chance de connaitre ce fameux « déclic », cette rencontre émotionnellement très marquante avec le livre qui conditionne tout leur avenir de lecteurs autonomes» (Navarro et Le Deun, 2004, p. 12). À la rive de leur vie d'adulte, en cinquième secondaire, il est nécessaire de travailler et de développer avec eux 
leur littératie et de leur faire comprendre comment il est possible d'imbriquer leurs apprentissages scolaires et l'ensemble de leurvie.

Ensuite, nous retrouvons les facteurs d'ordre social. Puisqu'ils sont peu valorisés à la maison, les actes de lire et d'écrire ne font pas partie de la vie quotidienne de certains élèves et donc, ils ne s'y intéressent pas, ou pas beaucoup, quand vient le temps de faire des tâches ou des activités en lien avec ces compétences à l'école. À long terme, cela les empêche d'enrichir l'apprentissage contextualisé, c'est-à-dire la croissance de la littératie par le contact avec des individus utilisant l'écrit pour des motifs fonctionnels (Giasson et Saint-Laurent, 1998). Un bon moyen de travailler cet aspect de la littératie avec les élèves est de mettre en place des discussions après la lecture d'une œuvre ou d'un texte et d'échanger sur celui-ci :

Les élèves tirent pleinement profit de la lecture quand ils peuvent réagir aux textes, y réfléchir et établir des rapprochements entre ce qu'ils ont lu et ce qu'ils ressentent et savent déjà, et Iorsqu'ils peuvent trouver de nouvelles idées, de nouvelles questions et de nouvelles informations dans leurs lectures (Gouvernement de l'Ontario, 2004).

Par ailleurs, Dumais (2011) note les facteurs culturels et rappelle qu'il est essentiel que l'enseignant tienne compte des référents culturels de chacun pour susciter l'intérêt notamment. Il doit également savoir diversifier les textes proposés aux élèves afin d'engager, de manière individuelle et collective, chaque élève culturellement. Les élèves doivent être capables de situer leur identité culturelle au sein de la classe, de la défendre, de l'expliquer et, surtout, d'être intéressés à comprendre celle des autres. L'enseignant doit « aider les élèves à comprendre d'autres modes de vie et influencer ainsi leur décision quant à la façon dont ils vont vivre dans le monde pluriel d'aujourd'hui » (Giasson, 2000, p. 8). Ainsi, notre matériel tient compte de ces facteurs puisqu'il propose différents textes qui permettent aux élèves d'échanger et de discuter selon leur bagage culturel (référents culturels, sociaux et familiaux), bien que nous travaillions dans une région où la pluralité des milieux se fait moins sentir qu'ailleurs.

Finalement, les facteurs d'ordre cognitif et métacognitif renvoient au fait que l'élève doit raisonner et se questionner lorsqu'il fait des activités d'enseignement-apprentissage (Dumais, 2011). Ceci amène l'enseignant à apprendre aux élèves à apprendre. Que ce soit par la régulation ou encore l'autorégulation, les élèves sont amenés à prendre conscience des stratégies qu'ils mettent en place pour décrire et comprendre un texte (Giasson, 2000). En échangeant avec leurs pairs sur la manière dont ils ont compris le texte et sur comment ils le perçoivent, les élèves apprennent par eux-mêmes. Et à cela vient s'ajouter, évidemment, la participation primordiale de l'enseignant qui observe et commente l'échange entre les élèves. Comme le souligne Dumais, « [d]ans la discipline du français, un moyen efficace de travailler l'aspect cognitif et métacognitif de la littératie est la lecture. En effet, le fait d'amener les élèves à réfléchir à leurs stratégies de lecture et à leur façon de comprendre et d'interpréter un texte leur permet de porter un regard sur les processus présents dans l'acte de lire » (2011, p. 6-7). 


\section{Méthodologie}

Le type de recherche que nous avons privilégié pour la création du matériel pédagogique est la recherche-développement. Selon Loiselle et Harvey (2007), ce type de recherche comporte deux parties distinctes, à savoir le développement d'interventions pédagogiques ainsi que l'émission des prescriptions qui guident les actions proposées dans les différentes activités créées. Le chercheur n'est pas seulement limité à ses besoins créateurs en concevant du matériel; il participe également à l'analyse de celui-ci afin de le peaufiner et de l'améliorer, et ce, après l'analyse d'experts (Loiselle et Harvey, 2007).

\section{Démarche suivie pour la mise sur pied du matériel pédagogique}

Nous désirions créer du matériel pédagogique afin de répondre à un besoin : celui d'enseigner la littératie aux élèves de la cinquième secondaire. Dans un premier temps, après avoir réalisé une recension des écrits, entre autres sur le concept de littératie, et parcouru des programmes d'enseignement, comme celui du MÉO (2004), nous en sommes venues à la conclusion qu'il est important de promouvoir son développement, et ce, même en cinquième secondaire. Travaillant dans une région défavorisée et pauvre culturellement, nous avons décidé de développer les compétences en littératie de nos élèves et peut-être, par le fait même, de changer leurs perceptions de leur propre langue. Ainsi, en valorisant à leurs yeux le savoir-lire, le savoir-écrire et le savoir-communiquer, par l'entremise de situations d'apprentissage et d'évaluation, en améliorant leurs compétences, nous sommes à même d'espérer qu'ils seront davantage en mesure de saisir la beauté de leur langue ainsi que toute l'importance du français pour leur avenir. Enfin, il semblait primordial que notre matériel permette aux élèves d'être actifs intellectuellement dans leurs apprentissages; les pratiques didactiques choisies, qui ne sont pas abordées dans le présent article, s'inscrivent donc dans les approches constructiviste et socioconstructiviste (notamment le cercle de lecture, l'enseignement explicite et la discussion). Dans un second temps, nous avons procédé à la création de nos différentes activités, de même qu'à la sélection des textes sur lesquels travailler avec les élèves. La recension des écrits réalisée précédemment constitue l'assise sur laquelle toutes nos activités sont basées. C'est l'ensemble de ces activités qui a été soumis à l'évaluation.

\section{Évaluation du matériel par les expertes}

Nous avons soumis notre matériel pédagogique à deux expertes : une enseignante de français en cinquième secondaire ainsi qu'une conseillère pédagogique. Les deux connaissent bien le milieu dans lequel nous enseignons. Nous trouvons pertinent qu'un membre actif dans notre école évalue notre travail sous un regard enseignant, de même que la conseillère pédagogique de notre commission scolaire, qui travaille toujours en étroite collaboration avec nous, et ce, dans plusieurs sphères de notre enseignement (formations, colloques, journées pédagogiques, mise sur pied de matériel, 
etc.). Les expertes sollicitées ont pu évaluer notre matériel pédagogique à l'aide d'un questionnaire, composé de huit questions à développement (par exemple, Selon vous, de quelle façon les activités du matériel pédagogique proposé sont en lien avec le programme de formation de l'école québécoise pour des élèves de la cinquième année du secondaire et en lien avec les approches constructiviste et socioconstructiviste?; Après avoir consulté le matériel pédagogique, êtes-vous en mesure de relever des points à améliorer? Si oui, lesquels?; Selon vous, est-ce que les activités sont complètes (explications, consignes et grilles claires, fiches d'activités contenant les informations importantes), et ce, tant pour l'enseignante que pour les élèves?).

Elles étaient donc invitées à faire ressortir les points forts et les points à améliorer, autant sur le contenu des activités que sur leur format. Elles pouvaient aussi émettre des commentaires généraux ou des suggestions. Pour chaque question, elles disposaient de plusieurs lignes pour noter et commenter le matériel, au fil de leur évaluation.

\section{Traitement des données}

Les données recueillies ont été analysées de manière qualitative. Nous avons conçu un tableau synthèse nous permettant de consigner les commentaires évaluatifs. Pour ce faire, nous avons créé les catégories suivantes, à savoir les points forts et les points à améliorer. Puis, nous avons consigné les propos dans différentes sous-catégories qui émergeaient de l'analyse des données. Pour les points forts, par exemple, les sous-catégo ries suivantes ont été créées : 1) originalité et pertinence du matériel; 2) respect du programme de formation de l'école québécoise; 3) souci pour les élèves en difficulté à travers le matériel; 4) littératie. Pour les points à améliorer, les sous-catégories suivantes ont été créées : 1) évaluation; 2) compétence « communiquer oralement selon des modalités variées »; 3) difficultés organisationnelles.

\section{Description du matériel pédagogique créé et évalué par les expertes}

Nous avons conçu six activités d'apprentissage et d'évaluation (SAÉ), dans lesquelles il est toujours possible d'évaluer les élèves ou, du moins, de leur donner des rétroactions constructives et de guider leurs apprentissages. Les activités construites touchent les trois compétences en français langue d'enseignement. Le matériel pédagogique créé comprend un tableau explicatif pour chaque activité, avec les trois phases d'une SAÉ (préparation, réalisation et intégration) ainsi que toutes les fiches nécessaires à sa réalisation et des fiches et des pistes liées à l'évaluation. Nous présentons en annexe quelques exemples des six activités créées.

Chacune de nos activités lie la lecture, l'écriture et l'oral; l'importance accordée à l'une ou l'autre des compétences varie cependant en fonction des activités. Celles-ci mettent également l'accent sur l'un ou l'autre des facteurs liés au développement de la littératie; encore une fois, la présence de chacun varie en fonction des activités. Grâce à plusieurs extraits de roman, la première activité 
proposée aux élèves, intitulée Le cercle de lecture, permet de travailler la lecture littéraire par l'entremise de la discussion et du cercle de lecture, de l'enseignement explicite, de la lecture interactive et d'une carte conceptuelle (schématisation des idées). La deuxième activité, Lecture à voix haute d'un album littéraire et conception d'une affiche littéraire, exploite un genre littéraire que nous oublions souvent au secondaire : l'album littéraire. Par l'entremise de cette activité, nous avons voulu faire redécouvrir le plaisir de lire les images aux élèves. Dans la troisième activité, Lecture et critique d'un album littéraire, les élèves sont appelés à se familiariser davantage avec le genre littéraire qu'est l'album, par l'entremise de la rédaction d'un résumé critique. Pour ce qui est de la quatrième activité, Tiroirs secrets, celle-ci propose aux élèves de cinquième secondaire d'agir comme des mini-profs pour les élèves de première secondaire. Dans la cinquième activité, La dernière cabane, nous avons voulu aborder la lecture par l'entremise d'une œuvre originale et novatrice, le livre de recettes de Martin Picard de la cabane à sucre Au Pied de Cochon et, plus particulièrement, par l'entremise de la nouvelle littéraire s'y retrouvant, écrite par Marc Séguin. La discussion, l'échange et le travail d'équipe sont au cœur de cette activité. Enfin, pour ce qui est de la sixième activité intitulée Tout est possible, celle-ci repose notamment sur la compétence orale (la discussion). En guise d'exemple, l'annexe 1 présente l'activité 2 , qui a notamment été soumise à l'évaluation.

\section{Résultats}

Dans cette partie, nous présentons une synthèse des commentaires formulés par les deux expertes. La première section présentera les points forts de notre matériel tandis que la deuxième sera consacrée aux points à améliorer.

\section{Points forts}

Nous allons traiter ici des points forts relevés par nos expertes: 1 ) originalité et pertinence du matériel; 2) respect du programme de formation de l'école québécoise; 3) souci des élèves en difficulté; 4) littératie.

\section{Originalité et pertinence du matériel}

Après avoir considéré les commentaires des expertes, nous dégageons le fait que notre matériel est original et pertinent. En effet, les deux expertes sont allées dans ce sens en précisant que le choix des œuvres dans l'ensemble des activités était non seulement très original, mais également pertinent pour des élèves de la cinquième secondaire. Les thèmes abordés (fréquentation, séduction, sexe, mort, environnement, nourriture, fatalité, etc.) rejoignent en effet une clientèle plus mature, presqu'adulte, et sauront intéresser et motiver les élèves plus âgés, les filles comme les garçons. Elles ont toutes les deux également souligné la diversité des activités et des genres littéraires proposés aux élèves (roman, nouvelle et album littéraire). Finalement, l'enseignante de la cinquième secondaire a mentionné le fait que les activités sont indépendantes les unes des autres et, qu'en ce sens, elles sont 
facilement réutilisables, adaptables ou, du moins, inspirantes pour un enseignant. « C'est original et créatif» mentionne, en conclusion, la conseillère pédagogique.

\section{Respect du programme de formation de l'école québécoise}

Ensuite, nous avons demandé aux expertes si le matériel proposé était toujours en lien avec le programme de formation de l'école québécoise et avec les approches constructiviste et socioconstructiviste, question à laquelle elles ont répondu positivement. En effet, d'un côté, la conseillère pédagogique a souligné le fait que les trois compétences sont touchées dans notre matériel. Elle affirme d'ailleurs que « l'une des spécificités du programme de 5e secondaire concerne la littérature et que nos activités sont en plein dans le mille! ». Elle mentionne également que les approches préconisées (constructivisme et socioconstructivisme) sont mises de l'avant et très bien exploitées tout au long des scénarios proposés, mettant l'apprenant au cœur de ses apprentissages.

L'enseignante de cinquième secondaire abonde dans le même sens et ajoute que « la littérature est un incontournable pour préparer la société de demain ». Elle mentionne que le matériel créé est adaptable tant aux élèves de niveau régulier qu'aux élèves aux prises avec des difficultés d'apprentissage, ce que prône le PFÉQ. L'enseignante relève d'ailleurs que le travail d'équipe planifié est un bon exemple qui prouve que nous avons à cœur l'interaction entre les élèves afin qu'ils s'entraident et, donc, que nous avons également à cœur leur réussite. De plus, les deux expertes ont relevé que la diversification des méthodes utilisées dans nos activités tend vers la construction des savoirs. En effet, la discussion, l'enseignement explicite et le cercle de lecture placent l'apprenant au centre de ses apprentissages, le rendant actif et curieux. Au regard de ces commentaires, nous pouvons donc affirmer que notre matériel respecte les attentes du programme de formation de l'école québécoise et qu'il met de l'avant les approches constructiviste et socioconstructiviste.

\section{Souci des élèves en difficulté}

Comme mentionné précédemment, nos activités permettent l'intégration des élèves en difficulté et peuvent très bien s'adapter en classe. La conseillère pédagogique a relevé le fait que nous accompagnions toujours de façon adéquate et motivante les élèves en difficulté et que des alternatives étaient souvent pensées pour eux. Elle a rappelé que la lecture à voix haute et l'utilisation de l'image sont d'excellents moyens pour soutenir un élève en difficulté, pour améliorer sa compréhension et son interprétation d'un texte ou d'une œuvre.

Par ailleurs, l'enseignante de la cinquième secondaire a rappelé que les trois étapes d'une SAÉ étaient toujours présentes, pertinentes et bien construites pour chacune des activités. La phase de préparation, notamment, permet toujours aux élèves de poser des questions et d'explorer le travail à faire par l'entremise d'une discussion ou d'une exploration de l'œuvre ou du texte étudié. "Ce temps est primordial lorsque nous enseignons à des élèves en difficulté puisqu'il permet de valider les acquis de chacun et de s'assurer de leur compréhension du travail demandé », rappelle l'enseignante. 
Les deux expertes ont également souligné l'importance de la phase de retour pour les élèves en difficulté ainsi que les rétroactions fréquentes de l'enseignant tout au long des différentes activités.

Finalement, les expertes ont dénoté le fait que les activités sont courtes dans le temps, ce qui est motivant pour des élèves plus faibles, et qu'elles permettent à ceux-ci de faire des choix, de créer, de coopérer, en plus d'être bien soutenu par l'enseignant.

\section{Littératie}

Finalement, les expertes ont mentionné le fait que notre matériel aborde la lecture littéraire et que c'est tout à fait à propos en cinquième secondaire. D'une part, la conseillère pédagogique a mentionné la pertinence et l'aspect motivationnel d'enseigner par l'entremise du caucus littéraire ainsi que du cercle de lecture. Elle affirme que le fait de vouloir faire vivre l'expérience esthétique lors de la lecture d'un texte ou d'une œuvre rejoint les critères d'évaluation des compétences en cinquième secondaire, notamment apprécier des textes variés et que cela permet aux élèves de s'ouvrir à euxmêmes, mais également sur le monde. L'enseignante a rappelé, quant à elle, qu'il est primordial de continuer de développer les compétences en littératie, même chez les élèves de la cinquième secondaire, puisque nous formons les citoyens de demain et que cela s'inscrit dans un processus continu.

\section{Points à améliorer}

Dans cette partie, nous allons mentionner les points à améliorer qu'ont relevés nos expertes : 1) évaluation; 2) compétence « communiquer oralement selon des modalités variées »; 3) difficultés organisationnelles.

\section{Évaluation}

Tout au long de leur lecture, les expertes commentaient les grilles d'évaluation et les façons d'évaluer les élèves. D’abord, elles ont toutes les deux ressorti le fait que nous devions évaluer les différents critères à l'aide d'un système de cote, au lieu des pourcentages. Nous avons rectifié le tir pour l'ensemble de nos activités. Aussi, l'enseignante de cinquième secondaire a mentionné l'absence de grille d'évaluation pour le scénario de l'activité 6 . Les consignes pour la création du scénario pourraient servir de balises pour construire une future grille d'évaluation, selon ce que l'enseignant désire évaluer chez les élèves.

Il nous a fallu également revoir certaines questions en lecture afin de nous assurer qu'elles appartenaient au bon critère d'évaluation. Enfin, à plusieurs endroits dans nos activités, nous suggérons désormais aux enseignants de conserver des traces du travail des élèves, qu'elles soient liées à l'écriture, à la lecture ou à la communication orale. 
Bien qu'elles aient apprécié nos façons d'aborder la compétence à communiquer oralement dans nos différentes activités, les deux expertes ont souligné le fait que nous aurions pu la développer davantage. La conseillère pédagogique a d'ailleurs suggéré de fréquemment émettre des rétroactions aux élèves sur la manière dont ils s'expriment à l'oral afin de les évaluer en contexte réel d'interaction avec les pairs et l'enseignant. Nous sommes de leur avis et ce pourrait être une piste intéressante et pertinente à exploiter dans l'adaptation de notre matériel.

\section{Difficultés organisationnelles}

Finalement, pour clore la section des points à améliorer, nous avons relevé les commentaires des expertes traitant des difficultés organisationnelles de nos activités. En effet, l'activité 4 , malgré le fait qu'elle soit des plus originales et que nous l'ayons révisée en profondeur, présente des lacunes sur le plan de la logistique et de la gestion du temps. Il est peu probable, en effet, que deux collègues de niveaux différents arrivent à s'entendre et à être sur la même longueur d'onde tout en ayant une planification et une gestion du temps similaire pour rendre cette activité réalisable dans un contexte réel d'enseignement. Nous croyons donc que cette activité dépend du contexte d'enseignement et professionnel, mais qu'elle est tout de même inspirante pour les enseignants.

\section{Limites du matériel pédagogique proposé et conclusion}

Tout d'abord, notre matériel, en version améliorée à la suite des commentaires des expertes, se doit de faire l'objet d'une expérimentation en classe afin de voir ses effets sur les élèves quant au développement de leurs compétences en littératie. L'évaluation du matériel, bien que nécessaire, ne saurait garantir hors de tout doute l'efficacité du matériel créé. En outre, le matériel a été mis sur pied en fonction de besoins spécifiques d'élèves issus d'un milieu en particulier; en ce sens, il ne saurait être généralisé ni employé tel quel. Il doit ainsi faire l'objet d'une adaptation par chaque enseignant. Nous avons pris la latitude de créer du matériel pédagogique susceptible d'intéresser la majorité des élèves de la cinquième secondaire, mais nous croyons qu'au final, ce sera à l'enseignant qui exploitera le matériel d'en juger. Par ailleurs, nous sommes conscientes que la dynamique motivationnelle diffère chez chaque élève et, donc, que ce ne sont pas toutes les activités proposées qui seront susceptibles d'intéresser tous les élèves. En effet, selon leurs valeurs, leurs intérêts et leurs humeurs, les élèves pourraient davantage être motivés par une activité que par une autre. Par exemple, nous croyons que le travail avec les albums pourrait moins intéresser certains élèves puisqu'ils croiraient que cela s'adresse à une clientèle plus jeune. Il leur faudra voir l'utilité de l'image et les éveiller à la matière. 
Néanmoins, bien que nous n'ayons pas été en mesure de présenter dans son intégralité le matériel amélioré à la suite de son évaluation, nous espérons que celui-ci trouvera preneur dans la communauté enseignante et, surtout, qu'il sera adapté et modifié pour permettre à plusieurs enseignants de se l'approprier et de développer la littératie chez leurs élèves. À moyen terme, il serait très pertinent de valider le matériel créé, et ce, dans différentes écoles et différents milieux scolaires ainsi qu'auprès de divers types d'élèves. 


\section{Références}

Bandura, A. (2003). Auto-efficacité. Le sentiment d'efficacité personnelle. Bruxelles : De Boeck.

Beauregard, F., Carignan, I. et Létourneau, M.-D. (2011). Recension des écrits scientifiques sur la littératie familiale et communautaire. Ministère de l'Éducation, du Loisir et du Sport (MELS). Repéré à https://depot.erudit.org/bitstream/003789dd/1/Beauregard_Carignan_MELS_litteratie_ familiale.pdf

Bibeau, R. (2009). Repéré à www.robertbibeau.ca/sae.html

Blaquière, S. (2012). Création d'un matériel pédagogique pour les élèves de 6e année du primaire permettant de développer les quatre compétences en français à l'aide de la littérature jeunesse (Mémoire de maitrise). Université de Sherbrooke.

Burns, S., Espinosa, L. et Snow, C. E. (2003). Débuts de la littératie, langue et culture: perspective socioculturelle. Revue des sciences de l'éducation, 29(1), 75-100. Repéré à http://id.erudit.org/ iderudit/009493ar

Dumais, C. (2011). La littératie au Québec : pistes de solution à l'école préscolaire et primaire. Repéré à http://www.forumlecture.ch/myUploadData/files/2011_2_Dumais.pdf

Giasson, J. et Saint-Laurent, L. (1998). Lire en classe : résultats d'une enquête au primaire. Université Laval. Revue canadienne de l'éducation, 24(2), 197-211.

Giasson, J. (2000). Les textes littéraires à l'école. Boucherville : Gaëtan Morin éditeur.

Gouvernement du Québec. (2007). Apprendre à lire : action concertée pour le soutien de la recherche en lecture. Québec: Ministère de l'Éducation, du Loisir et du Sport (MELS). Repéré à www.mels. gouv.qc.ca/stat/recherche/app_fr.pdf

Gouvernement de l'Ontario. (2004). La littératie au service de l'apprentissage : Rapport de la Table ronde des experts en littératie de la $4^{e}$ à la $6^{e}$ année. Toronto : Ministère de l'Éducation de l'Ontario. Repéré à http://www.edu.gov.on.ca

Lebrun, J., Lenoir, Y. et Desjardins, J. (2004). Le manuel scolaire « réformé » ou le danger de l'illusion du changement: analyse de l'évolution des critères d'évaluation des manuels scolaires de l'enseignement primaire au Québec entre 1979 et 2001. Revue des sciences de l'éducation, XXX(3), 509-533.

Legendre, M.-F. (2012). Jean Piaget et le constructivisme en éducation. Dans C. Gauthier et M. Tardif (dir.), La pédagogie, Théories et pratiques de l'Antiquité à nos jours (p. 203-214). Montréal : Gaëtan Morin éditeur. 
Lenoir, Y., Roy, G. R., Rey, B. et Lebrun, J. (2001). Le manuel scolaire et l'intervention éducative, regards critiques sur ses apports et ses limites. Sherbrooke : Éditions du CRP.

Loiselle, J. et Harvey, S. (2007). La recherche développement en éducation : fondements, apports et limites. Recherches qualitatives, 27(1), 40-59.

Masciotra, D. (2007). Le constructivisme en termes simples. Vie pédagogique, 143, 48-52.

Makdissi, H., Boisclair, A. et Sirois, P. (2010). La littératie au préscolaire : une fenêtre ouverte vers la scolarisation. Québec, Québec: Presses de l'Université du Québec.

Navarro, H. et Le Deun, E. (2004). Prévenir l'illettrisme: Une autre approche pour reconstruire la lecture. Paris: Éditions Magnard.

Piaget, J. (1974). La prise de conscience. Paris : PUF.

Royer, N. (1995). Éducation et intervention au préscolaire. Montréal: Gaëtan Morin éditeur.

Vygotsky, L. S. (1985). Pensée et langage. Paris : Éditions sociales.

Zakhartchouk, J.-M. (1999). L'enseignante, un passeur culturel. Paris : E.S.F.

\section{Bibliographie des œuvres littéraires exploitées dans le matériel pédagogique}

Bernard, F. et Roca, F. (2004). L'indien de la tour Eiffel. Belgique : Éditions du Seuil.

Leblanc, L. (1999). 371⁄2AA. Montréal : Éditions TYPO et Louise Leblanc.

Pellerin, G. (1982). Filature. Nuit blanche, magazine littéraire, (7), 30-31.

Pennac, D. (1987). La fée carabine. Paris, France : Éditions Gallimard.

Séguin, M. (2012). La dernière cabane. Montréal : Restaurant au Pied de cochon..

Vian, B. (1996). Et on tuera tous les affreux. Paris : Fayard et Le Livre de Poche.

Werber, B. (2002). L'Arbre des possibles et autres histoires. Paris : Éditions Albin Michel.

Xabi, M., et Thiébaut, O. (2008). Tiroirs secrets. Paris : Éditions Sarbacane. 


\section{Annexe 1}

Voici l'activité 2 proposé dans notre matériel pédagogique visant le développement de la littératie en cinquième secondaire par l'entremise de l'album littéraire.

\section{Activité 2 : Lecture à voix haute d'un album littéraire et conception d'une affiche littéraire}

\begin{tabular}{|c|c|c|}
\hline $\begin{array}{l}\text { Titre de l'activité : Lecture à voix haute d'un } \\
\text { album littéraire et conception d'une affiche } \\
\text { littéraire }\end{array}$ & Tâches de l'enseignante : & Tâches des élèves: \\
\hline & \multicolumn{2}{|l|}{ 1. PRÉPARATION } \\
\hline $\begin{array}{l}\text { Durée approximative : } \\
4 \text { périodes de } 75 \text { minutes }\end{array}$ & $\begin{array}{l}\text { 1.1 Dans une présentation PowerPoint, l'ensei- } \\
\text { gnante présente l'album littéraire L'indien de } \\
\text { la tour Eiffel de façon générale aux élèves ainsi } \\
\text { qu'un bref aperçu de son auteur Fred Bernard } \\
\text { et de son illustrateur, François Roca. L'album } \\
\text { circule en classe pendant ce temps (la page } \\
\text { couverture et la quatrième de couverture sont } \\
\text { cachées par du papier). }\end{array}$ & $\begin{array}{l}\text { 1.1 Les élèves écoutent attentivement la } \\
\text { présentation de l'enseignante. Ils peuvent la } \\
\text { questionner à tout moment. }\end{array}$ \\
\hline $\begin{array}{l}\text { Approches pédagogiques : } \\
\text { - Lecture à voix haute; } \\
\text { - Lecture interactive; } \\
\text { - Imagerie mentale; } \\
\text { - Discussion. }\end{array}$ & $\begin{array}{l}\text { 1.2 L'enseignante montre ensuite aux élèves, } \\
\text { via Google, différentes affiches littéraires qui } \\
\text { servent souvent à promouvoir la vente d'une } \\
\text { œuvre littéraire en librairie (style, originalité, } \\
\text { image, titre, jeux de mots, mots-clés, etc.). } \\
\text { Elle peut y aller avec des œuvres du moment } \\
\text { ou demander aux élèves des titres de livres et } \\
\text { chercher leur affiche littéraire sur Internet. Elle } \\
\text { leur montre que c'est souvent à cause d'une } \\
\text { question publicitaire et que nous achetons une } \\
\text { œuvre parce que l'affiche est belle, intéres- } \\
\text { sante, simple ou complexe et qu'elle suscite } \\
\text { notre curiosité, nos intérêts (comme nous } \\
\text { le faisons, par exemple, au simple regard de } \\
\text { la page couverture d'une œuvre). Le tout se } \\
\text { déroule sous forme de discussion et l'ensei- } \\
\text { gnante invite les élèves à y participer. À noter } \\
\text { que l'affiche littéraire est souvent une reprise } \\
\text { de la page couverture d'une œuvre. }\end{array}$ & $\begin{array}{l}\text { 1.2 Les élèves écoutent l'enseignante et com- } \\
\text { mentent les affiches littéraires qu'elle présente. } \\
\text { Ils peuvent notamment dire pourquoi tel ou } \\
\text { tel élément retient davantage leur attention, } \\
\text { leur intérêt. Ils participent à la discussion et ils } \\
\text { peuvent questionner l'enseignante. }\end{array}$ \\
\hline $\begin{array}{l}\text { Compétences et composantes spécifiques } \\
\text { touchées en français langue d'enseigne- } \\
\text { ment : Écrire des textes variés: } \\
\text {-Faire appel à sa créativité (explorer différentes } \\
\text { façons d'aborder un sujet, susciter l'intérêt de } \\
\text { différents destinataires). }\end{array}$ & $\begin{array}{l}\text { 1.3 L'enseignante distribue la feuille d'écoute } \\
\text { en lien avec l'expérience esthétique aux élèves. } \\
\text { Elle leur explique celle-ci en les questionnant } \\
\text { sur l'expérience esthétique créée par l'œuvre } \\
\text { (les atmosphères dans l'histoire et les senti- } \\
\text { ments et réactions du lecteur), notion qui ne } \\
\text { leur sera pas étrangère. Il s'agit d'une portion } \\
\text { plus théorique, mais que les élèves auront déjà } \\
\text { travaillée. Elle rappelle qu'ils peuvent consulter } \\
\text { leurs notes de cours pour plus d'informations } \\
\text { sur cette notion. }\end{array}$ & $\begin{array}{l}\text { 1.3 Les élèves écoutent l'enseignante et la } \\
\text { questionnent, si nécessaire. Ils peuvent valider } \\
\text { leurs connaissances antérieures sur l'expé- } \\
\text { rience esthétique en posant des questions ou } \\
\text { en émettant des commentaires. Les élèves } \\
\text { ne remplissent pas tout de suite la feuille } \\
\text { d'écoute, mais elle doit leur servir de guide } \\
\text { pour brièvement noter les différents éléments } \\
\text { de l'expérience esthétique des affiches litté- } \\
\text { raires sur une feuille mobile. }\end{array}$ \\
\hline
\end{tabular}




\begin{tabular}{|c|c|c|}
\hline $\begin{array}{l}\text { Titre de l'activité : Lecture à voix haute d'un } \\
\text { album littéraire et conception d'une affiche } \\
\text { littéraire }\end{array}$ & Tâches de l'enseignante : & Tâches des élèves: \\
\hline $\begin{array}{l}\text { Communiquer oralement selon des moda- } \\
\text { lités variées : } \\
\text { - Construire du sens (planifier son écoute, } \\
\text { comprendre et interpréter des communica- } \\
\text { tions orales, y réagir). } \\
\text { - Réfléchir à sa pratique d'auditeur, d'inter- } \\
\text { locuteur et de locuteur (évaluer l'efficacité } \\
\text { de ses stratégies d'écoute et de prise de } \\
\text { parole). }\end{array}$ & $\begin{array}{l}\text { 1.4 Pendant quelques minutes, l'enseignante } \\
\text { invite les élèves à échanger en équipes de } \\
\text { quatre ou cinq sur les affiches présentées et à y } \\
\text { puiser les différents éléments esthétiques. Elle } \\
\text { circule en classe. }\end{array}$ & $\begin{array}{l}\text { 1.4 Les élèves, en équipes de quatre ou cinq, } \\
\text { échangent sur les différentes affiches et sur les } \\
\text { éléments qui appartiennent à l'expérience es- } \\
\text { thétique. Ils s'aident de leurs notes de cours et } \\
\text { peuvent questionner l'enseignante. Ils notent } \\
\text { le tout sur une feuille mobile. }\end{array}$ \\
\hline $\begin{array}{l}\text { Genre littéraire exploité : } \\
\text { Album littéraire : L'indien de la tour Eiffel } \\
\text { (auteur: Fred Bernard; illustrateur: François } \\
\text { Roca). }\end{array}$ & \multirow[t]{2}{*}{$\begin{array}{l}\text { 1.5 En grand groupe, sous forme de discus- } \\
\text { sion, l'enseignante invite les élèves à faire une } \\
\text { mise en commun des éléments trouvés. Elle } \\
\text { procède affiche par affiche et elle s'assure du } \\
\text { droit de parole de toutes les équipes. }\end{array}$} & \multirow[t]{2}{*}{$\begin{array}{l}\text { 1.5 Les élèves participent à la mise en com- } \\
\text { mun en grand groupe. Ils peuvent poser des } \\
\text { questions et faire des commentaires en lien } \\
\text { avec les affiches littéraires présentées et la } \\
\text { notion d'expérience esthétique. }\end{array}$} \\
\hline $\begin{array}{l}\text { Matériel requis: } \\
\text { Album littéraire, feuilles mobiles, notes de } \\
\text { cours, carton blanc ou de couleur, crayons, } \\
\text { local informatique, images, ciseau, colle. }\end{array}$ & & \\
\hline
\end{tabular}

\begin{tabular}{|c|c|}
\hline Tâches de l'enseignante : & Tâches des élèves : \\
\hline \multicolumn{2}{|l|}{ 2. RÉALISATION } \\
\hline $\begin{array}{l}\text { 2.1 L'enseignante annonce aux élèves qu'ils devront écouter la lecture } \\
\text { de l'album littéraire (par l'enseignante) et réaliser ensuite une affiche } \\
\text { littéraire pour en faire la publicité. Ils ne verront pas la page couverture } \\
\text { ni la quatrième de couverture de l'œuvre, qui servent très souvent de } \\
\text { référents dans ce genre littéraire. Pour réaliser leur affiche, ils pour- } \\
\text { ront choisir d'exploiter un ou plusieurs éléments reliés à l'expérience } \\
\text { esthétique. Elle leur rappelle qu'ils devront être sélectifs dans le choix } \\
\text { des éléments exploités pour ne pas surcharger leur affiche. Ils peuvent } \\
\text { utiliser le dessin ou le collage d'images (support papier ou numérique). }\end{array}$ & $\begin{array}{l}\text { 2.1 Les élèves écoutent attentivement les consignes du travail à faire. Ils } \\
\text { peuvent questionner l'enseignante pour des précisions. }\end{array}$ \\
\hline $\begin{array}{l}2.2 \text { L'enseignante fait la lecture à voix haute de l'album. Elle demande } \\
\text { aux élèves de remplir la feuille d'écoute sur l'expérience esthétique pour } \\
\text { y consigner des éléments importants pendant la lecture. }\end{array}$ & $\begin{array}{l}\text { 2.2 Les élèves écoutent attentivement la lecture de l'album par l'en- } \\
\text { seignante. Ils sont aussi en mode « prise de notes active » à l'aide de } \\
\text { la feuille d'écoute. Après la lecture, ils peuvent compléter la feuille et y } \\
\text { ajouter des éléments. }\end{array}$ \\
\hline $\begin{array}{l}\text { 2.3 L'enseignante invite les élèves à former des équipes de deux ou de } \\
\text { trois, au maximum. Attention! Même s'ils sont trois, les élèves doivent } \\
\text { s'assurer d'être concis. C'est la qualité et l'originalité de l'affiche qui } \\
\text { prévaudront sur la quantité d'informations. Elle rappelle qu'ils ont deux } \\
\text { périodes en classe pour créer leur affiche. Au cours de la dernière pé- } \\
\text { riode, ils présenteront leur travail aux autres (pas de temps prédétermi- } \\
\text { né). L'enseignante circule en classe, aide et conseille les élèves. }\end{array}$ & $\begin{array}{l}\text { 2.3 En équipe de deux et de trois, au maximum, les élèves font une mise } \\
\text { en commun des différentes notes qu'ils ont prises pendant l'écoute de } \\
\text { l'album et ils amorcent la réalisation de leur affiche littéraire. Ils peuvent } \\
\text { utiliser leurs notes de cours et ils ont accès au local informatique. Leur } \\
\text { affiche peut se faire autant par collage papier ou numérique que par } \\
\text { dessin (possibilité de projet avec un enseignant d'arts plastiques). }\end{array}$ \\
\hline
\end{tabular}




\begin{tabular}{|c|c|}
\hline Tâches de l'enseignante : & Tâches des élèves: \\
\hline \multicolumn{2}{|l|}{ 3. INTÉGRATION } \\
\hline $\begin{array}{l}\text { 3.1 L'enseignante rappelle que la présentation à la classe est informelle. } \\
\text { Elle invite les élèves à former un cercle avec leurs chaises et à s'assoir } \\
\text { avec leurs coéquipiers. Donc, en grand groupe, elle invite les élèves à } \\
\text { présenter leur affiche littéraire et à justifier leurs choix d'images ou de } \\
\text { dessins. Elle les invite à faire des liens avec leur expérience esthétique. } \\
\text { Il est à noter que seule l'affiche sera évaluée, mais les élèves doivent } \\
\text { tout de même justifier leurs choix d'éléments, d'images et d'informa- } \\
\text { tions qu'ils ont mis sur leur affiche (points de participation à l'oral). } \\
\text { L'enseignante guide les présentations des élèves et commente celles-ci. } \\
\text { Elle attire l'attention sur certains éléments de leurs affiches et invite les } \\
\text { élèves à réagir, à commenter. }\end{array}$ & $\begin{array}{l}\text { 3.1 Les élèves écoutent l'enseignante et ils présentent ensuite à la } \\
\text { classe leur choix d'images et de dessins pour représenter l'album litté- } \\
\text { raire. Ils justifient tel ou tel élément et les informations choisies et mis } \\
\text { en valeur dans leur affiche littéraire. Les élèves participent activement à } \\
\text { la présentation des affiches. }\end{array}$ \\
\hline $\begin{array}{l}\text { 3.2 L'enseignante montre enfin l'ensemble de l'album littéraire aux } \\
\text { élèves, la page couverture et la quatrième de couverture. Elle leur } \\
\text { demande s'ils auraient inclus d'autres éléments sur leur affiche s'ils } \\
\text { avaient vu l'ensemble de l'album. Si oui, lesquels et pourquoi? Leur } \\
\text { écoute et leur prise de notes auraient-elles été différentes? Comment? } \\
\text { Pourquoi? }\end{array}$ & $\begin{array}{l}\text { 3.2 Les élèves écoutent les questions de l'enseignante et prennent } \\
\text { connaissance de l'ensemble de l'album littéraire. Ils participent à la } \\
\text { discussion en grand groupe et échangent avec l'enseignante. }\end{array}$ \\
\hline $\begin{array}{l}\text { Il est à noter que les affiches des élèves seront exposées en classe ou } \\
\text { dans le corridor. Les élèves pourront voter pour la meilleure affiche } \\
\text { littéraire, par un vote secret en classe. }\end{array}$ & $\begin{array}{l}\text { À la fin du cours, les élèves affichent leur travail sur un mur de la classe } \\
\text { ou dans le corridor. Les élèves pourront voter par un vote secret pour la } \\
\text { meilleure affiche littéraire. }\end{array}$ \\
\hline
\end{tabular}

